\title{
Giant cell arteritis: a real life evaluation of service quality at the Royal National Hospital for Rheumatic Diseases, Bath
}

\author{
Authors: Thomas Williams, ${ }^{A}$ Diluka Karunaratne ${ }^{B}$ and Sarah Skeoch ${ }^{C}$
}

\section{Introduction}

Giant cell arteritis (GCA) is the most common form of systemic vasculitis with an incidence of 41 to 113 cases per 100,000 people aged over 50 years in North American and European populations, affecting women approximately 1.5 times more frequently than men. ${ }^{1}$ GCA is characterised by ischaemic complications including acute-onset visual loss in approximately $20 \%$ of patients. ${ }^{2}$ Late recognition and treatment of GCA increases the risk of such adverse events, hence the need for prompt diagnosis. High dose corticosteroids, given over a prolonged period, remain the mainstay of GCA treatment, despite the recent National Institute for Health and Care Excellence (NICE) approval of the IL-6 inhibitor tocilizumab for refractory cases. ${ }^{3,4}$ Such treatment is itself associated with significant morbidity, including increased risk of osteoporosis, diabetes, infection and cardiovascular disease, hence the importance of avoiding over-diagnosis and inappropriate treatment. Both of these perils emphasise the need for safe and effective systems of referral, triage and assessment of suspected GCA.

While temporal artery biopsy remains the gold standard diagnostic test for GCA, there is a high rate of false negative results due to the discontinuous distribution of large vessel inflammation and following steroid treatment. Temporal artery ultrasound is increasingly being used to support diagnosis given its non-invasive nature and superior sensitivity to biopsy prior to steroid treatment, as well as in detecting relapse of GCA 5. Its performance as a diagnostic test is highly operator dependent and its sensitivity falls rapidly with treatment, hence is best used in a high volume centre by expert sonographers, as early as possible after treatment is initiated, or prior to treatment where feasible. Alternative imaging options include PET-CT, MR angiography or CT angiography, all of which are usually favoured for extra-cranial disease. EULAR guidelines now recommend that where a high pre-test probability for GCA exists and high quality imaging findings support that diagnosis, there is no need to perform further tests. ${ }^{5}$ Equally, a low pre-test probability and negative imaging is felt to be sufficient

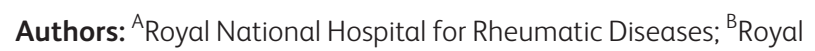
United Hospitals, Bath; ${ }^{C}$ Royal National Hospital for Rheumatic Diseases, Bath to exclude the diagnosis, though in all other cases, further efforts should be made towards establishing a definitive diagnosis, such as a temporal artery biopsy. ${ }^{5}$

The Royal National Hospital for Rheumatic Diseases (RNHRD) in Bath is a tertiary rheumatology centre and is staffed by a team including (but not limited to) consultants, trainee doctors and nursing staff, with a vast amount of experience in managing rheumatological conditions. RNHRD has a fast track referral pathway, which can be accessed by local general practitioners (GPs), in order to facilitate the rapid assessment of suspected giant cell arteritis urgently on the day case unit by a specialty registrar, aiming for review within 3 working days of referral, always performed in normal working hours Monday to Friday. Newly suspected GCA referrals are not therefore seen in routine outpatient appointments and there is no formal capacity limit on the number of referrals seen. Where appropriate and capacity allows, the assessing registrar may request a temporal artery ultrasound from the vascular imaging team at the nearby Royal United Hospital in Bath, and/or a temporal artery biopsy to be performed as day case surgery by the local ophthalmology team, in order to support diagnosis. Patients diagnosed with GCA are subsequently followed up in general outpatient clinics as there is no dedicated clinic for GCA.

The aim of our study was to evaluate the quality of the RNHRD GCA service by measuring against agreed quality standards, patient and GP feedback, in order to identify any areas which could be further improved.

\section{Materials and methods}

Four separate prospective data collection exercises were performed concurrently over a 2-month period from 24 September 2018 to 23 November 2018: a) an audit of all newly suspected GCA patients attending for initial assessment at RNHRD as day cases; b) an audit of all patients with an established diagnosis of GCA attending for outpatient follow-up appointments at RNHRD; c) a satisfaction questionnaire of patients attending for their initial day case assessment; d) an online survey of local GPs.

Parts $a$ and $b$ were collected using standardised data collection forms, which was designed after team consultation and review of the relevant NICE, Royal College of Physicians, European League Against Rheumatism (EULAR) and British Society of Rheumatology (BSR) recommendations. 
Part c was a paper-based questionnaire which asked patients whether they felt they had received a clear explanation of the diagnosis and management plan, would know who and when to contact if their symptoms changed and asked for any suggestions for service improvement. This survey was issued to all patients being assessed for suspected GCA by the ward clerk of the RNHRD day case unit, not only those given a GCA diagnosis.

Part d was a GP survey issued electronically through the 'Survey Monkey' website to 64 local general practices, allowing one response per practice. This asked GPs four questions: whether they were aware of the fast track GCA assessment service at RNHRD; whether they knew how to access this service; whether they felt the discharge summaries from these assessments provide the right amount of information; whether there is any other aspect of the GCA service that they feel could be improved.

\section{Results and discussion}

During the 2-month period of data collection, 30 patients were newly assessed for suspected GCA on the day case unit at RNHRD, of whom 15 were diagnosed with GCA based on clinical assessment, ie pre-ultrasound ( $50 \%)$. The mean and median delay from referral by the GP to assessment at RNHRD was 2 days, even allowing for fallow days eg on weekends. In 21 (70\%) cases a temporal artery ultrasound was requested, which occurred on average 0.4 days after being requested and 3.5 days after steroid treatment had been initiated. The ultrasound demonstrated findings consistent with GCA in eight cases, though in five of these GCA had not been deemed the most likely cause of symptoms on clinical assessment. None of these patients subsequently received a temporal artery biopsy in order to clarify the diagnosis, despite EULAR recommendations. In one case where there was felt to be a high clinical suspicion for GCA but negative ultrasound findings after 2 days of treatment, a temporal artery biopsy confirmed a GCA diagnosis.

Over the same period, 34 patients were followed up in various RNHRD outpatient clinics for a primary diagnosis of GCA. The time from diagnosis to this assessment ranged from 6 to 587 weeks. Of these, 16 (47\%) had either a confirmatory biopsy or consistent imaging to support the clinical diagnosis. Only 9 patients had received follow-up appointments at all the time intervals recommended by BSR guidelines. ${ }^{6}$

Fifteen patients provided feedback on their care. All were satisfied with the explanations offered to them and felt well enough informed about how to escalate any subsequent concerns. One patient commented that they were assessed in a chair as the day case unit lacked sufficient beds at that time. Anecdotally this is a fairly common experience, though no other patients commented on this issue. Seventeen GP surgeries completed the electronic survey of the 64 invited. 13/17 (76\%) were aware of the existence of the RNHRD fast track GCA assessment service, of whom 10/17 (59\%) answered that they'd know how to refer to it. Some of the suggestions for improvement mentioned that more could be done to ensure GPs were given the correct information about how to refer these cases, including by the hospital appointments team.

These results demonstrate that whilst RNHRD provide a good level of service which allows timely assessment of patients with suspected GCA, supported by vascular ultrasound, more can be done to eliminate some of the inconsistencies in the care provided; to ensure sufficient bed space, nursing and medical capacity; and to inform local GPs about how to access the service in order to avoid inappropriate over and under diagnosis. To this end, the following improvements should be considered prior to re-evaluation of the GCA service.

> Education sessions for trainee rheumatology doctors, GPs, ophthalmologists, vascular team and other relevant clinicians about the most recent evidence base in GCA, including the importance of obtaining a timely temporal artery ultrasound and how this should be interpreted.

> Supporting business cases for the vascular imaging and ophthalmology teams to ensure adequate capacity for timely temporal artery ultrasound and biopsies.

$>$ Ensuring all administrative staff involved in booking outpatient appointments can appropriately re-direct GCA referrals from GPs to the fast track assessment pathway instead.

\section{Conclusions}

RNHRD currently offer a fast track assessment service for newly suspected GCA, including access to vascular ultrasound where appropriate, with a mean delay from referral to assessment of 2 days. This helps facilitate prompt and accurate diagnosis in order to avoid the pitfalls of both under and over treatment of this condition. This audit demonstrates that most patients referred receive high quality care, with broadly positive feedback from patients and GPs, though more needs to be done to highlight this service to some local GPs and to secure the capacity of both the rheumatology and vascular imaging services to cope with future demand.

\section{References}

1 Buttgereit F, Dejaco C, Matteson EL et al. Polymyalgia and giant cell arteritis: a systematic review. JAMA 2016;315:2442-58.

2 Dasgupta B (on behalf of the RCP Giant Cell Arteritis Guideline Development Group). Concise guidance: diagnosis and management of giant cell arteritis. London: RCP, 2010.

3 Tamaki H, Hajj-Ali RA. Tocilizumab for giant cell arteritis - a new giant step in an old disease. JAMA Neurol 2018;75:145-6.

4 National Institute for Health and Care Excellence. Tocilizumab for treating giant cell arteritis. Technology appraisal guidance [TA158]. London: NICE, 2018.

5 Dejaco C, Ramiro S, Duftner C et al. EULAR recommendations for the use of imaging in large vessel vasculitis in clinical practice. Ann Rheum Dis 2018;77:636-43.

6 Dasgupta B, Borg FA, Hassan N et al. BSR and BHPR guidelines for the management of giant cell arteritis. Rheumatology (Oxford) 2010;49:1594-7. 